\title{
Multislice Simulation of Dynamical Elastic Scattering and Orbital Angular Momentum of Vortex Beams in Crystals
}

\author{
Lin $\mathrm{Xie}^{1}$, Peng Wang ${ }^{1}$ and Xiaoqing Pan ${ }^{1,2}$ \\ 1. National Laboratory of Solid State Microstructures and College of Engineering and Applied sciences, \\ Nanjing University, Nanjing 210093, People's Republic of China \\ 2. Department of Materials Science and Engineering, University of Michigan-Ann Arbor, Ann Arbor \\ America.
}

In analogy to optical vortices, electron beam carrying quantized orbital angular momentum (OAM) has been realized both on transmission electron microscope (TEM) [1] and scanning TEM (STEM) recently, which opened a new path to spin and magnetic structure characterizations at nano or even atomic scale using energy-loss magnetic chiral dichroism (EMCD)[1-3]. The dynamical elastic scattering in the conventional atomic resolution STEM imaging[4] or spectrum mapping[5] of a crystalline specimen on zone axis has been studied in depth, which showed that the image contrast is strongly dependent on the channeling effect of the beam along atomic columns. As a new form of electron beam, the dynamical elastic scattering of the vortex beam in crystals is yet to be studied and understood fully. It is still an open question that how the well-defined OAM of a vortex beam can be affected by the dynamical elastic scattering during the propagation inside crystals, which could be important for the quantitative interpretation of EMCD signal. In this paper, we study the dynamical elastic scattering of the vortex beam in a crystal using multislice simulation and demonstrate the dependence of the OAM of the propagating vortex beam on the atomic number $Z$, projected atomic potential and the sample thickness.

In our multislice simulation[6], a crystal model of non-spinpolarized cubic perovskite $\mathrm{BiFeO}_{3}$, which consists of high-, medium- and low- $Z$ elements, is built and viewed along [001] direction. An atomic scale vortex beam carrying OAM $l=+1$ is generated with accelerating voltage of $300 \mathrm{kV}$, convergence semi-angle of $25 \mathrm{mrad}$ and free of aberrations. Fig. 1 (a)-(c) show the calculated propagation of the beam intensity inside the crystal when the beam is focused on the entrance surface above the $\mathrm{Bi}, \mathrm{Fe}-\mathrm{O}$ and $\mathrm{O}$ columns, respectively. We can see that the vortex beam focused on $\mathrm{Bi}$ or Fe-O columns strongly channels along the atomic columns and its characteristic doughnut shape and size of the beam is preserved even up to a thickness of $20 \mathrm{~nm}$, which suggests that the helical phase front of the vortex beam is kept unchanged. This electron channeling effect can be phenomenologically explained by the coupling between the incident vortex beam and the $2 p$ bound states of the projected atomic potential [7], in analogy to the $1 s$ state channeling for the conventional STEM beam [8]. On the O column, however, as shown in Fig. 1(c), the vortex beam quickly diverges and becomes delocalized. Such unexpected delocalization can be readily explained by the weak coupling between the probe and the $2 p$ state of oxygen as oxygen is a low- $Z$ element.

Furthermore, the expected values $\left\langle J_{z}>\right.$ of the vortex beam along different atomic columns as a function of thickness are also calculated. Surprisingly, $\left\langle J_{z}\right\rangle$ is highly $Z$-dependent as shown in Fig. 2 . On the Bi column, $\left\langle J_{z}>\right.$ oscillates in the range of $0 \sim 13 \mathrm{~nm}$ but remains positive up to a thickness of $40 \mathrm{~nm}$. On the $\mathrm{Fe}-\mathrm{O}$ or $\mathrm{O}$ column, however, a reversal of $\left\langle J_{z}\right\rangle$ occurred at a thickness of about $32 \mathrm{~nm}$ and $27 \mathrm{~nm}$, respectively. This result is beyond our expectation as the $\left\langle J_{z}\right\rangle$ of the vortex beam along atomic column 
of low- $Z$ element will reverse its OAM at a thinner thickness. It should also be noted that the reversal of $\left\langle J_{z}\right\rangle$ is highly unfavorable as it may complicate the interpretation of inelastic scatterings in the application of EMCD.

In conclusion, the dynamical elastic scattering and OAM of a vortex beam in a crystal are investigated using multislice simulation method. Our results show that the atomic number $Z$, projected atomic potential and the thickness should be taken into careful consideration for quantitative interpretations of the inelastic scatterings of vortex beams.

References:

[1] J Verbeeck, H Tian and P Schattschneider, Nature 467 (2010), p. 301.

[2] J Verbeeck et al, Applied Physics Letter 99 (2011), 203109.

[3] J Verbeeck, H Tian and A Béché, Ultramicroscopy 113 (2012), p. 83.

[4] P D Nellist and S J Pennycook, Ultramicroscopy 78 (1999), p. 111.

[5] L J Allen et al, Physical Review Letters 91 (2003), 105503.

[6] E J Kirkland in "Advanced Computing in Electron Microscopy", (Springer) p. 198.

[7] Huolin L Xin and H M Zheng, Microscopy and Microanalysis 18 (2012), p. 711.

[8] J Broeckx, M Op de Beeck and D Van Dyck, Ultramicroscopy 60 (1995), p. 71.
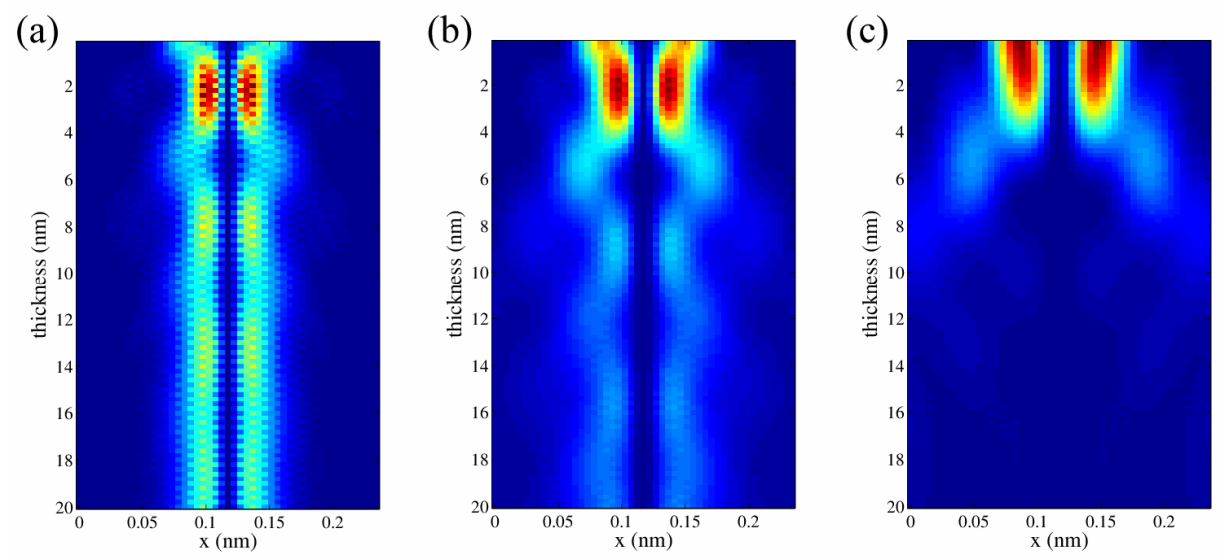

Figure 1. Multislice simulations of the intensity distribution of a vortex beam focused on the entrance surface above (a) Bi column, (b) Fe-O column and (c) O column, respectively.

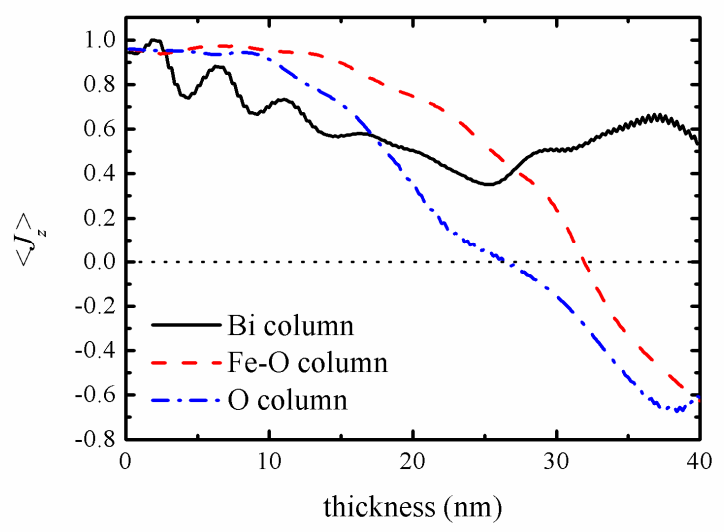

Figure 2. $\left\langle J_{z}>\right.$ of vortex beam as a function of sample thickness at $\mathrm{Bi}, \mathrm{Fe}-\mathrm{O}$ and $\mathrm{O}$ column. 